\title{
Controle inteligente aplicado à um sistema de bombeamento de água
}

\author{
H. A. M. MOREIRA*. J. M. VillanUeVA**, H. P. GoMeS ${ }^{* * * *}$ \\ *Departamento de Engenharia Mecânica, Universidade Federal da Paraíba, \\ João Pessoa, Brasil, (Tel: $81996041599 ;$ e-mail: HugoMarinho93@outlook.com) \\ **Departamento de Engenharia Elétrica, Universidade Federal da Paraíba, \\ João Pessoa, Brasil, (e-mail: Jmauricio@cear.ufpb.br) \\ ***Departamento de Engenharia Civil, Universidade Federal da Paraíba, \\ João Pessoa,Brasil, (e-mail: Heberp@uol.com.br)
}

\begin{abstract}
This work aimed at the development of an intelligent controller based on the Fuzzy architecture with the Mandani inference system and Gaussian membership functions, with a small number of rules, to control the pressures of a simulated supply system in the form of experimental bench in the LENHS/UFPB. The controller was developed through the relationships between the outputs and inputs of the system and operator expertise, implemented in the LABVIEW platform and interconnected to the plant through a SCADA system Texas Instruments. The control is performed under a frequency inverter that varies the rotation of a pump. As input data are the measurements of the three pressure transducers and that of a flow meter installed in the discharge line of the CMB (Moto Motor - Pump). The results show the effective control of the pressures, with maximum overtaking and reasonable accommodation time.
\end{abstract}

Resumo: Este trabalho teve como objetivo o desenvolvimento de um controlador inteligente baseado na arquitetura Fuzzy com o sistema de inferência Mandani e funções de pertinência gaussianas, com um reduzido número de regras, para o controle das pressões de um sistema de abastecimento simulado na forma de bancada experimental no LENHS/UFPB. O controlador foi desenvolvido através das relações entre as saídas e entradas do sistema e da expertise do operador, implementado na plataforma LABVIEW e interligado à planta através de um sistema SCADA da Texas Instruments. O controle é realizado sob um inversor de frequência que varia a rotação de uma bomba. Como dados de entrada estão as medições dos três transdutores de pressão (PT) e a de um medidor de vazão (FT) instalados na linha de descarga do CMB (Conjunto Moto - Bomba). Os resultados mostram o controle efetivo das pressões, com máxima ultrapassagem e tempo de acomodação considerados satisfatórios.

Keywords: Fuzzy Systems; Energy efficiency; Pressure control; Water supply.

Palavras-chaves: Sistemas nebulosos; Eficiência energética; Controle de pressões; Abastecimento de água.

\section{INTRODUÇÃO}

As técnicas computacionais progrediram muito nos últimos anos e estão tomando o lugar da matemática clássica na modelagem e no projeto de controladores para sistemas de controle. De certo, sistemas muito complexos e com não linearidades severas são melhores representados por modelos computacionais, principalmente por modelos inteligentes. A lógica Fuzzy, ou também, lógica nebulosa foi proposta inicialmente por Mandani (1976), baseada nos conjuntos Fuzzy propostos por Zadeh (1965). Ela é uma técnica de controle bem particular, na qual permite o modelamento do pensamento ou da expertise humana de forma simples e intuitiva. Tal técnica é robusta o suficiente para lidar com imprecisões, não-linearidades e até alterações temporais sutis no sistema. Devido a sua fácil aplicação, a lógica Fuzzy vem sendo amplamente utilizada, como nos trabalhos de Barcelos et al. (2017), que desenvolveu um estudo sobre a aplicação da lógica Fuzzy em um processo de absorção de $\mathrm{CO} 2 \mathrm{em}$ uma torre de absorção que apresenta altas não-linearidades. Assim como no trabalho desenvolvido por Sousa et al. (2017) que se concentrou no desenvolvimento de um controlador Fuzzy multivariável para um processo de produção de gás liquefeito. Ambos trabalhos tiveram seus controladores Fuzzies comparados a controladores convencionais do tipo PID, apresentando sempre desempenhos superiores. Neves et al. (2017), por sua vez, realizou uma avaliação experimental de um sistema fotovoltaico de modo a garantir a estabilidade. Seixas e Corrêa (2018) desenvolveram um controlador Fuzzy sintonizado por algoritmos genéticos (AG) para o controle da carga energética em um sistema de supercapacitores. Os resultados mostram melhor desempenho do controlador Fuzzy sintonizado frente ao controlador Fuzzy convencional, isso porque o AG tem como função otimizar os intervalos das funções de pertinência.

Outros trabalhos também foram desenvolvidos visando a aplicação da lógica Fuzzy em sistemas de bombeamento e abastecimento como os de Lavor (2012), Oliveira (2012), Salvino (2016), Mendonça (2016) e Barros Filho et al (2017) que a utilizaram com foco na eficiência energética de sistemas 
de bombeamento e distribuição de água, entretanto, os controladores projetados possuíam um elevado número de regras e de funções de pertinência. Ainda que amplamente utilizada, poucos estudos foram realizados acerca da estrutura Fuzzy e da sua base de inferência, como os de Sugeno (1985), Sugeno e Kang (1986), Mandani (1976). Além disso, não existem estudos concretos que tratem do estudo das Funções de Pertinências (MF's) e como elas devem ser aplicadas à cada sistema. Escolhendo-se normalmente as funções triangulares e as trapezoidais.

Este trabalho tem como objetivo principal projetar e implementar um controlador baseado na lógica FuzzyMandani, com funções de pertinência gaussianas e um reduzido número de regras, em comparação com as funções triangulares e trapezoidais, na plataforma LABVIEW para o controle de pressões de uma planta de abastecimento, simulada em forma de bancada experimental, através da variação da velocidade do CMB.

\section{LÓGICA FUZZY}

Reconhecida como a melhor ferramenta para se trabalhar com sistemas do tipo "caixa preta", a lógica Fuzzy é muito eficiente em situações nas quais é preciso modelar o raciocínio humano. Segundo Ross (2005) ela é utilizada em casos nos quais a formulação do modelo matemático do sistema é complexo ou impossível. Um controlador baseado em técnicas Fuzzy contém, ao menos, três componentes segundo Mandani (1976):

- Dicionário, que define conjuntos Fuzzy sobre as variáveis (Fuzzyficação);

- Base de regras, que estabelece uma relação entre as variáveis de entrada e de saída;

- Método de inferência, usado para determinar a saída dado uma certa entrada.

Eventualmente, pode-se acrescentar uma quarta componente, chamada de Defuzzyficação, que transforma uma saída fuzzy em um número real ou um conjunto clássico.

Na Fig. 1 a arquitetura de um sistema de inferência FuzzyMandani é mostrado. Inicialmente as variáveis de entradas são Fuzyficadas - variável numérica é transformada em variável linguística - e agrupadas em um determinado conjunto linguístico. As regras que são do tipo "Se-Então", são consultadas na etapa de inferência. "As regras Fuzzy descrevem situações específicas que podem ser submetidas à análise de especialistas, e cuja inferência conduz a algum resultado desejado" (Ortega, 2001).

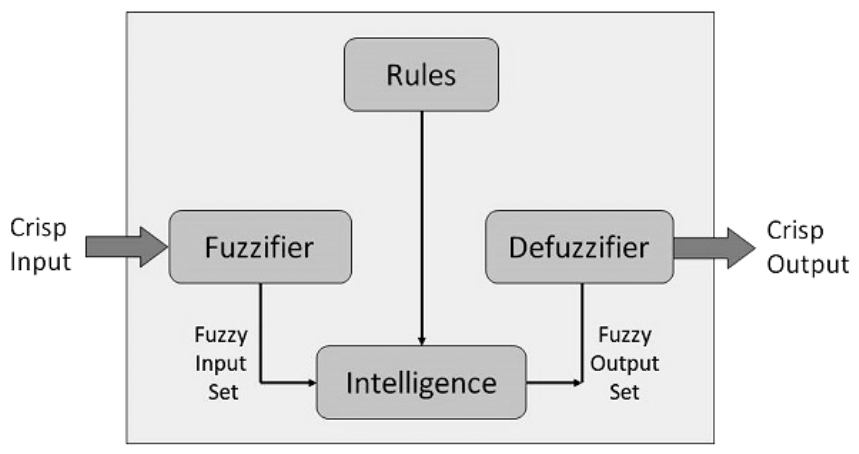

Figura 1. Arquitetura Fuzzy-Mandani. Disponível: www.tutorialspoint.com.

\section{SISTEMAS DE ABASTECIMENTO}

Segundo Gomes (2012) sistemas de abastecimento de água é o conjunto de equipamentos, obras e serviços voltados para o suprimento de água à comunidade, para fins de consumo doméstico, industrial e público. Sua concepção e dimensionamento ocorrem de forma integrada, o que requer, geralmente, o emprego de uma equipe de profissionais especializados. Na Fig. 2 é mostrado um sistema de abastecimento simplificado desde à captação da água até a sua distribuição ao consumidor final, a etapa final é concluída através da impulsão da água por uma estação de bombeamento.

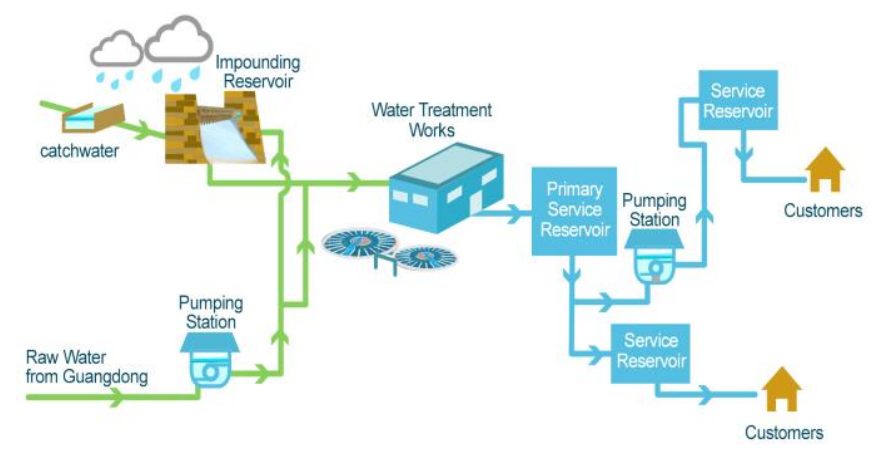

Figura 2. Sistema de abastecimento de água. Disponível: https://www.wsd.gov.hk.

Tais sistemas consomem uma grande quantidade de energia elétrica, ainda segundo Gomes (2012), os sistemas de abastecimento e de esgotamento sanitário são responsáveis por, aproximadamente, $3 \%$ da energia consumida no mundo, sendo o sistema de bombeamento responsável por até $97 \%$ desse montante, o que faz com que métodos de redução do consumo energético sejam utilizados, entre eles a automação do sistema por meio de sistemas de controle inteligente.

\section{PROJETO DOS CONTROLADORES}

\subsection{Sistema de Controle}

Na Fig. 3 é mostrado um típico sistema de controle incremental MISO (Multiple Input Single Output). Onde y é a saída, $e(\mathrm{k}) \mathrm{e}$ $e(\mathrm{k}-1)$ são o erro atual e anterior, respectivamente, $y^{*}$ é o set point, $f(\mathrm{k})$ e $f(\mathrm{k}-1)$ são os sinais de frequência, $\Delta f$ é o gradiente de frequência e $d$ é o distúrbio inserido no sistema.

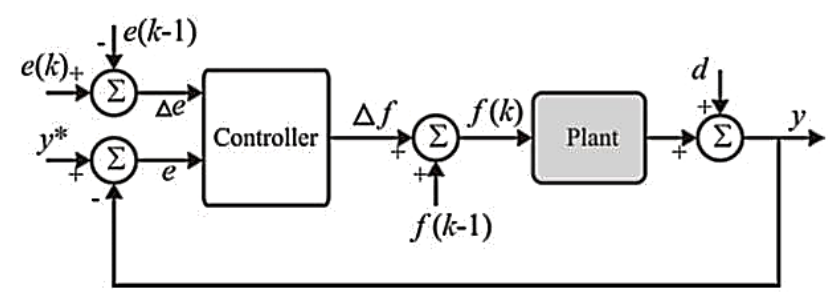

Figura 3. Sistema de controle MISO.

Este tipo de arranjo é bastante utilizado em sistemas industriais. Neste trabalho o distúrbio é inserido por uma válvula manual ou eletrônica (VRP), a depender do 
experimento realizado. A ação da válvula altera a dinâmica do sistema, deslocando a curva da tubulação na posição vertical e exigindo que o controlador atue para controlar a pressão no set-point desejado através de um diferencial de frequência, ou seja, alterando a rotação do CMB (Conjunto Motor-Bomba). A curva da bomba também é deslocada verticalmente com a alteração da sua rotação, de modo a encontrar o equilíbrio com a curva da tubulação.

\subsection{Controlador Fuzzy}

O projeto do controlador Fuzzy se dá através da observação do sistema e da inter-relação de suas entradas e saídas. Deste modo, as variáveis de entradas foram o erro e a variação do erro da pressão (PT-05) e a variável de saída é o delta de frequência. Os conjuntos Fuzzies de entrada foram definidos como: Negativo Grande (NG), Zero (Z) e Positivo Grande (PG). Já os conjuntos Fuzzies de saída foram Decremento Grande (DG), Zero (Z) e Incremento Grande (IG) de acordo como mostra a Fig. 4.

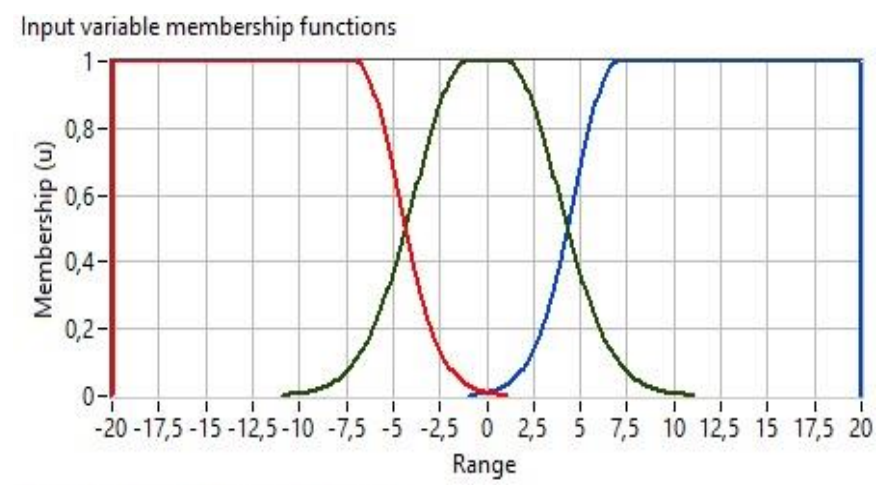

Input variable membership functions

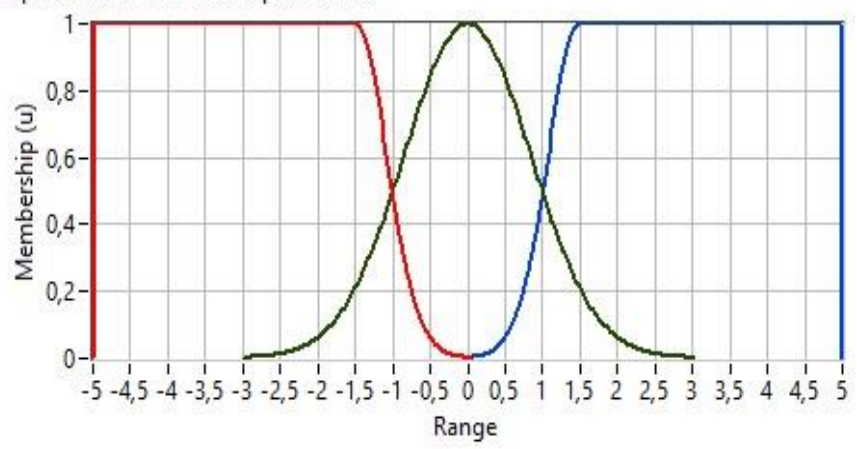

Output variable membership functions

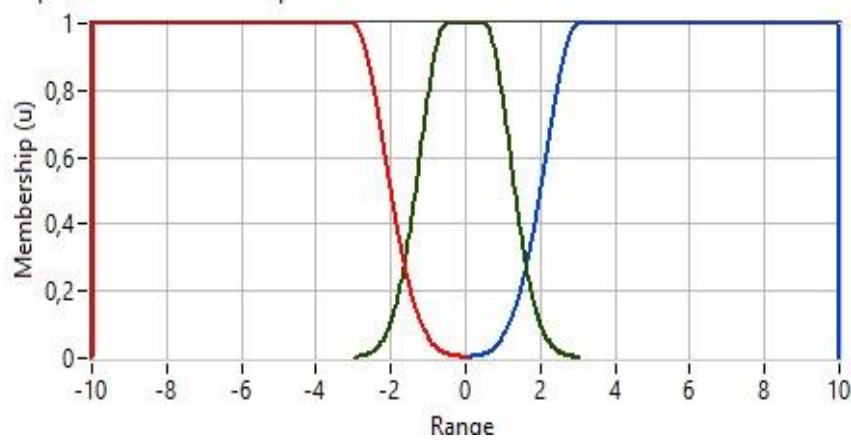

Figura 4. Conjuntos e MF Gaussianas do controlador Fuzzy.
Tabela 1. Conjunto de regras para o controlador Fuzzy.

\begin{tabular}{|c|c|c|c|c|}
\hline \multirow{3}{*}{ Erro } & \multicolumn{4}{|c|}{ Variação do erro } \\
\hline & & NG & Z & PG \\
\cline { 2 - 5 } & NG & IG & IG & Z \\
\cline { 2 - 5 } & $\mathrm{Z}$ & IG & Z & DG \\
\cline { 2 - 5 } & PG & Z & DG & DG \\
\hline
\end{tabular}

O conjunto de regras mostrado na Tabela 1 em conjunto com os conjuntos e as Funções de Pertinência (MF's) mostradas na Figura 4, resultam na superfície de controle mostrada na Figura 5.

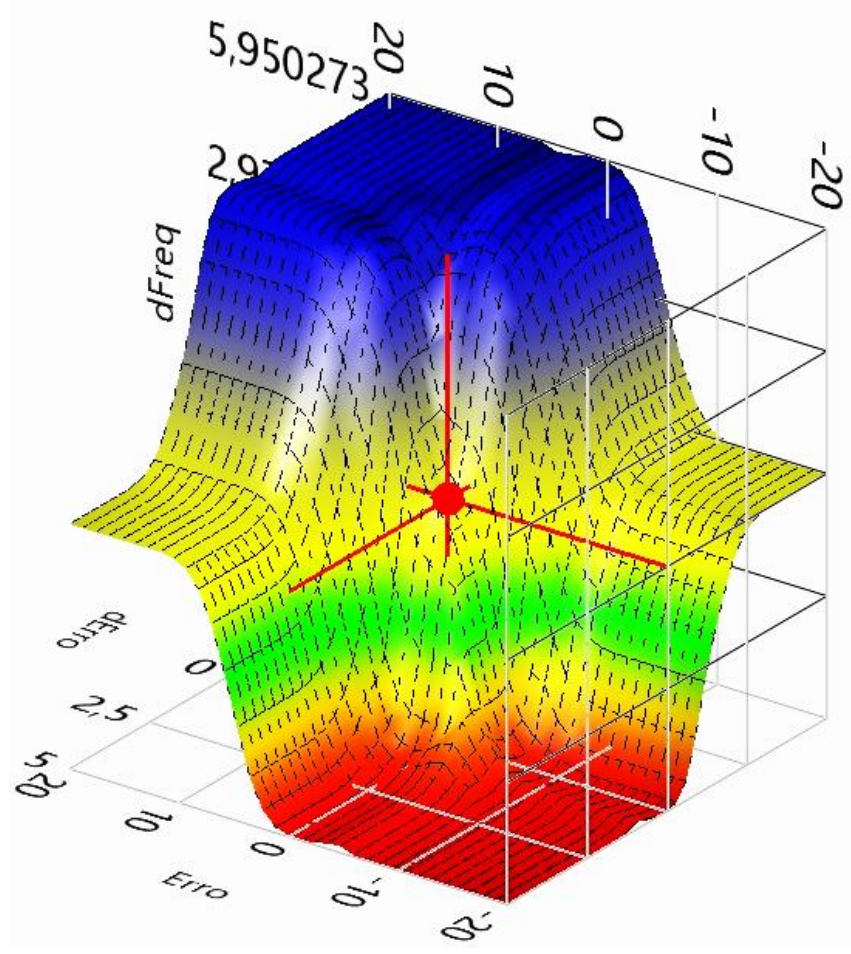

Figura 5. Superfície do controlador Fuzzy.

\section{SISTEMA EXPERIMENTAL}

Este trabalho contou com uma bancada experimental mostrada na Fig. 6 -, que está alocada no Laboratório de Eficiência Energética e Hidro Sanitária da Universidade Federal da Paraíba (LENHS/UFPB). Ao fundo e a direita é possível observar o sistema SCADA da Texas Instruments (NI USB-6229). No canto direito da Fig. 6 estão o painel de força e os dois inversores de frequência: um CFW-11 da WEG e um Altivar 31 da Schneider Electric. Os dois CMB são da DANCOR e possuem 5 HP cada. Além disso, podem operar em arranjo série/paralelo através da abertura e do fechamento manual de válvulas. Há também uma Válvula Redutora de Pressão (VRP) eletrônica e comandada através do supervisório na plataforma LABVIEW, ela tem como função variar o consumo de água e, consequentemente, as pressões de serviço para que o controlador atue mantendo as pressões nos setpoints desejados. O sensoriamento ficam a cargo de cinco 
transdutores de pressão (Druck PTX 7217) e dois transdutores de vazão eletromagnéticos (VMS Pro 038), bem como, de dois sinais enviados pelos inversores de frequência para a leitura da potência ativa dos CMB's e um enviado pela VRP para indicar o seu ângulo de abertura. Entretanto, neste trabalho foi utilizada apenas parte da bancada: três sensores de pressão, um medidor de vazão, um CMB, um inversor de frequência (CFW-11) e a VRP.

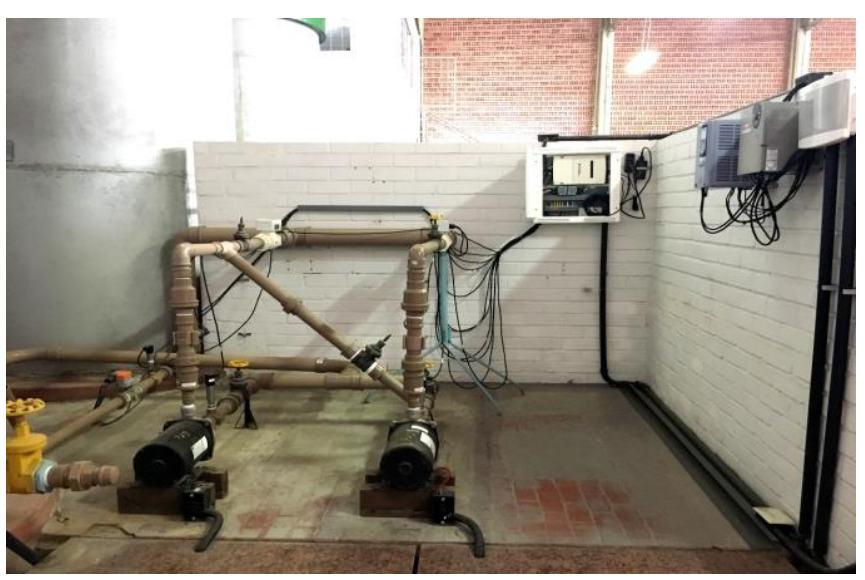

Figura 6. Bancada experimental no LENHS/UFPB.

\section{RESULTADOS}

\subsection{Resposta Transitória}

Os resultados apontam o controle da pressão de serviço nos set points desejados. Além disso, o tempo de subida é razoável e ficou dentro dos limites aceitáveis para o sistema $(\mathrm{t}=3,7$ segundos), bem como o erro de regime permanente $(e=0,53 \%)$, como pode ser visto na Fig. 7. Uma ressalva é feita ao realizar testes com pressões acima de 12,5 metros de coluna d'agua (m.c.a.), o sensoriamento apresenta grande variância motivada pela abertura involuntária da válvula eletrônica.

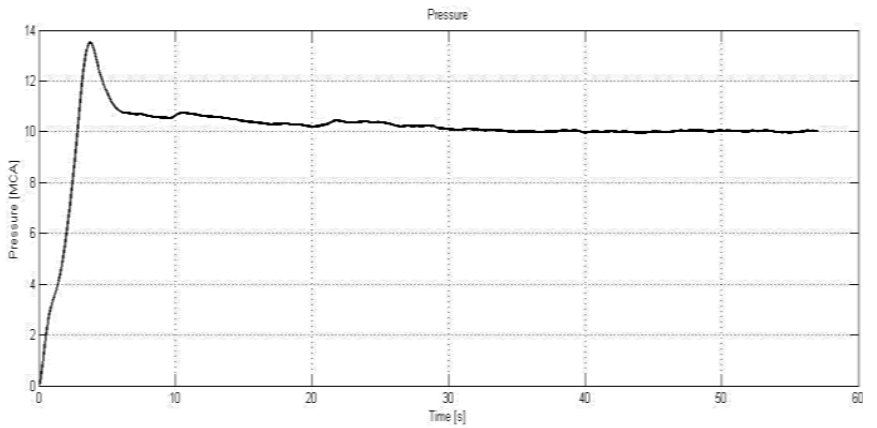

Figura 7. Resposta degrau com abertura da VRP em $40^{\circ}$ e setpoint em 10 m.c.a.

Um outro ensaio foi realizado de forma a avaliar a resposta transitório variando o set-point do controlador. Os set-points foram definidos em 5, 7,5, 10 e 12,5 m.c.a. A partir de 12,5 m.c.a. a pressão controlada entra em regime oscilatório, isso ocorre devido a abertura involuntário da válvula eletrônica (VRP), entretanto, para as demais pressões o comportamento é satisfatório.

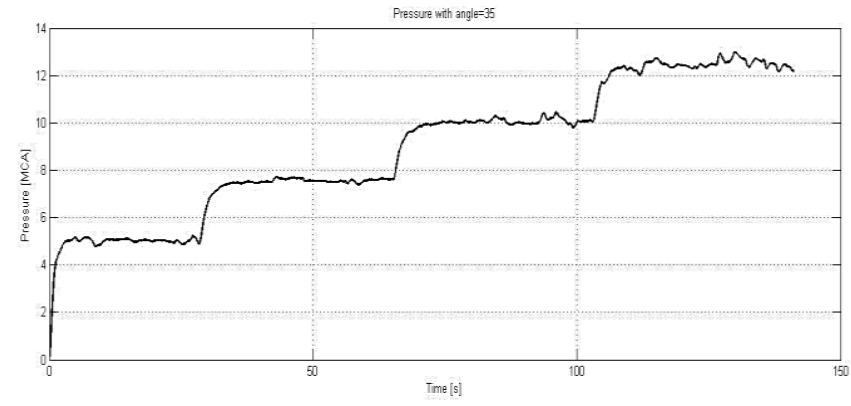

Figura 8. Variação do set-point do controlador com abertura da VRP em 35.

\subsection{Análise da Estabilidade}

A análise da estabilidade do controlador se deu através do fechamento, em velocidade constante, da VRP, partindo de um ângulo de $30^{\circ}$ até $70^{\circ}$, quando ela se encontra parcialmente fechada. A análise da pressão controlada, mostrada na Fig. 9, ressalta a robustez do controlador, mantendo a pressão com um erro menor que $10,5 \%$.

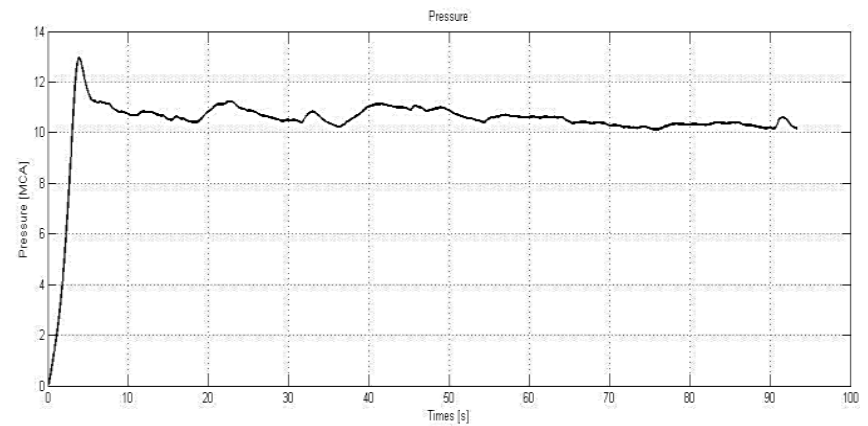

Figura 9. Análise da estabilidade do controlador com o fechamento da VRP e set-point em 10 m.c.a.

\subsection{Comparação entre Controladores}

Para efeito comparativo, o gráfico da Fig. 10 expõe a comparação entre um controlador com MF's triangulares com 49 regras (linha contínua) com o controlador desenvolvido neste trabalho (linha pontilhada). O controlador com um menor número de regras apresenta uma máxima ultrapassagem maior e isto já era esperado. Entretanto, o erro de regime permanente é menor $0,57 \%$ contra $1,33 \%$, do controlador com MF's triangulares.




Figura 10. Comparação entre controlador de 9 regras gaussiano e 49 regras triangular.

\section{CONCLUSÕES}

Este trabalho tratou da utilização da lógica Fuzzy, utilizando MF's gaussianas com um reduzido número de regras em comparação com funções triangulares e um elevado número de regras, para o controle de um sistema de bombeamento visando o controle das pressões de serviço e, consequentemente, a redução do consumo elétrico. Os objetivos almejados foram atingidos, o controlador se mostrou robusto o suficiente para controlar distúrbios do tipo degrau (realizados através do fechamento ou da abertura total e brusca da válvula de bloqueio). Além disso, a redução do consumo elétrico se mostrou consistente. Trabalhos futuros poderão tratar do estudo sobre o LCC (Life Cycle Cost) do CMB, já que uma vez reduzido a rotação dos motores poderá haver uma redução significativa dos custos de manutenção dos equipamentos, bem como o aumento da sua vida útil.

\section{AGRADECIMENTOS}

Os agradecimentos são direcionados à CAPES/CNPQ pela bolsa de mestrado concebida e ao LENHS/UFPB pela figura de seu coordenador e meu orientador Heber Pimentel Gomes.

\section{REFERÊNCIAS}

Barros Filho, E. G.; Salvino, L. R.; Bezerra, S. T. M.; Salvino, M. M.; Gomes, H. P. Intelligent system for control of water distribution networks. Water Science \& Technology: Water Supply, p. 1-12, 2017.

Barcelos, A. S. Barcelos, M. S. Bertini, L. Copetti, A. Modelagem e simulação de controladores fuzzy para o controle do processo de absorção de co2. Simpósio Brasileiro de Automação Inteligente, 2017.

Carvalho, P. S. O. Controle para bombeamento distribuído com vistas à minimização dos custos energéticos aplicado a sistemas de abastecimento de água, 2012. Tese (Doutorado em Engenharia Mecânica) - Programa de Pós Graduação em Engenharia Mecânica, UFPB, João Pessoa, PB, 2012.

Mamdani, E. H. (1977). Application of fuzzy logic to approximate reasoning using linguistic synthesis, IEEE Transactions on Computers C-26(12): 1182-1191.

M. Sugeno, Industrial Applications of Fuzzy Control, Elsevier, New York, 1985.

Mendonça, K. H. Sistema de controle fuzzy na operação otimizada de redes setorizadas com bombeamento direto, 2016. Dissertação (Mestrado em Engenharia Mecânica) Programa de Pós Graduação em Engenharia Mecânica, UFPB, João Pessoa, PB, 2016.

Neves, C. C. Medeiros, R. L. P. Ayres, F. A. C. Barra, W. Yoshino, F. K. Avaliação experimental de desempenho de controlador fuzzy aplicado a um sistema de foto geração de energia elétrica. Simpósio Brasileiro de Automação Inteligente, 2017.
Ross, T. J. Fuzzy logic with engineering applications. John Wiley and Sons, 2005.

Salvino, L. R. Eficiência energética em sistemas pressurizados de distribuição de água com o uso de redes neurais artificiais, 2016. Tese (Doutorado em Engenharia Mecânica) - Programa de Pós Graduação em Engenharia Mecânica, UFPB, João Pessoa, PB, 2016.

Seixas, L. D. Corrêa, F. C. Controlador fuzzy sintonizado por algoritmo genético em sistema de armazenamento de energia. Simpósio de Mecânica Computacional, 2018.

Souza, A. L. A. Pires, A. H. M. Araújo, F. M. U. Junior, J. M. A. Controlador fuzzy multivariável aplicado ao processo de produção do gás liquefeito de petróleo. Simpósio Brasileiro de Automação Inteligente, 2017.

Sugeno, M.; Kang, G. T. (1986). Structure identification of fuzzy model. Fuzzy Sets and Systems, 28:329-346.

Oliveira, J. K. C. Lógica fuzzy aplicada na otimização operacional de rede setorizada de distribuição de água. 2017. 147f. Tese (Doutorado em Engenharia Mecânica) Programa de Pós Graduação em Engenharia Mecânica, UFPB, João Pessoa, PB, 2017. 